\title{
Construction and Expected Performance of the Hadron Blind Detector for the PHENIX Experiment at RHIC
}

\author{
A Milov ${ }^{1}$, W Anderson ${ }^{4}$, B Azmoun ${ }^{1}$, C-Y Chi ${ }^{2}$, A Drees ${ }^{3}$, \\ A Dubey ${ }^{4}$, M Durham ${ }^{3}$, Z Fraenkel $^{4}$, J Harder $^{1}$, T Hemmick $^{3}$, \\ R Hutter ${ }^{3}$, B Jacak ${ }^{3}$, J Kamin ${ }^{3}$, A Kozlov ${ }^{4}$, M Naglis ${ }^{4}$, \\ P O'Connor ${ }^{1}$, R Pisani ${ }^{1}$, V Radeka ${ }^{1}$, I Ravinovich ${ }^{4}$, \\ T Sakaguchi ${ }^{1}$, D Sharma ${ }^{4}$, A Sickles ${ }^{1}$, S Stoll $^{1}$, I Tserruya ${ }^{4}$, \\ B Yü ${ }^{1}$, C Woody ${ }^{1}$ \\ ${ }^{1}$ Brookhaven National Laboratory, Upton, NY 11973-5000, USA \\ ${ }^{2}$ Nevis Columbia Laboratories, Irvington, NY 10533, USA \\ ${ }^{3}$ Stony Brook University, SUNY, Stony Brook, NY 11794, USA \\ ${ }^{4}$ Weizmann Institute, Rehovot 76100, Israel \\ E-mail: amilov@bnl.gov
}

\begin{abstract}
A new Hadron Blind Detector (HBD) for electron identification in high density hadron environment has been installed in the PHENIX detector at RHIC in the fall of 2006. The HBD will identify low momentum electron-positron pairs to reduce the combinatorial background in the $e^{+} e^{-}$mass spectrum, mainly in the lowmass region below $1 \mathrm{GeV} / \mathrm{c}^{2}$. The $\mathrm{HBD}$ is a windowless proximity-focusing Cherenkov detector with a radiator length of $50 \mathrm{~cm}$, a CsI photocathode and three layers of Gas Electron Multipliers (GEM). The HBD uses pure $\mathrm{CF}_{4}$ as a radiator and a detector gas. Construction details and the expected performance of the detector are described.
\end{abstract}

\section{Physics goal and detector concept}

One of the primary goals of the PHENIX [1] experiment at RHIC is to study the production of low-mass electron-positron pairs [2] as a tool to investigate the properties of the new state of matter discovered at RHIC. Results of similar studies published by CERN experiments [3, 4] at lower energy show an excess of $e^{+} e^{-}$pairs produced in the mass region below $1 \mathrm{GeV} / \mathrm{c}^{2}$. The main difficulty to a measurement in this mass region is the combinatorial background from the $e^{+} e^{-}$pairs with a small opening angle coming from the $\pi^{0}$-meson decays and $\gamma$-conversions. To overcome this problem the PHENIX experiment designed and built a new detector to reject such $e^{+} e^{-}$pairs.

The detector concept is described in [5]. The HBD is a proximity-focusing windowless Cherenkov counter. Pure $\mathrm{CF}_{4}$ is used as the radiator gas and the amplification media. This choice has several advantages crucial for the detector 
operation. A high refraction index $(n=1.00062$ in the visible region and higher in the UV) allows production of more Cherenkov photons than most other gases. $\mathrm{CF}_{4}$ is fully transparent up to $11.5 \mathrm{eV}$ which makes it a very good match to the CsI photocathode with $>70 \%$ quantum efficiency in the deep UV. The electron extraction efficiency into $\mathrm{CF}_{4}$ is among the highest of any gas; e.g. more than a factor of 2 higher than that into Ar. $\mathrm{CF}_{4}$ based mixtures are used as working gas in many detectors, including PHENIX. We have shown [7, 8] that pure $\mathrm{CF}_{4}$ can be used as a detector gas.

The HBD is located close to the interaction vertex. It starts after the beam pipe at a radius of $5 \mathrm{~cm}$ and extends up to an outer radius of $65 \mathrm{~cm}$. The HBD is made of two identical arms. An exploded view of one detector arm is shown in Fig. 1. A
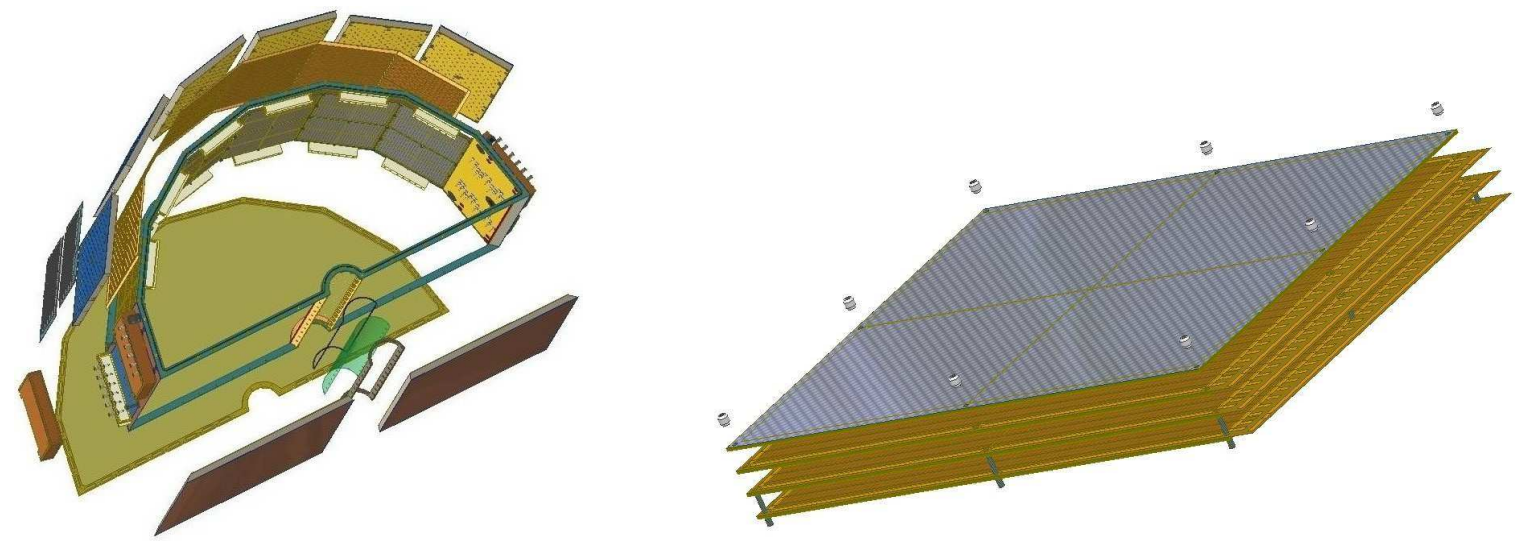

Figure 1. Left: Exploded view of the HBD detector. One side panel is removed for clarity. Right: A photodetector module consisting of 3 GEMs and a mesh.

thin FR4 frame holds panels made of FR4/Honeycomb/FR4 $(250 \mu \mathrm{m} / 19 \mathrm{~mm} / 250 \mu \mathrm{m})$ sandwiches. The 2 larger front panels and the 8 smaller back panels are glued to a frame that provides rigidity to the box. The two side panels (the closer one is removed for clarity) are mounted with plastic screws. Among the 8 back panels the outer 2 are reserved for the detector services such as gas in/out, high voltage, UV windows and the 6 inner panels are equipped each with 2 photodetector modules on the inside and connected to the Front End Electronics (FEE) board attached to the outer surface of the detector. Electrons produced in the collision enter the detector through a $127 \mu \mathrm{m}$ mylar window coated with $100 \mathrm{~nm}$ of $\mathrm{Al}$ and radiate Cherenkov photons along their path in $50 \mathrm{~cm}$ of $\mathrm{CF}_{4}$ radiator.

The photodetector shown in the right panel of Fig. 1 is a stack of 3 GEMs $27 \times 22 \mathrm{~cm}^{2}$ stretched on a $1.5 \mathrm{~mm}$ thick and $5 \mathrm{~mm}$ wide FR4 frame. A cross-like $0.3 \mathrm{~mm}$ wide support runs in the middle of the frame. The top GEM facing the detector volume has a 0.2-0.4 $\mu \mathrm{m}$ layer of CsI evaporated on its surface also plated with gold to prevent CsI poisoning by the $\mathrm{Cu}$ of the GEM. A $90 \%$ transparent stainless steel mesh $1.5 \mathrm{~mm}$ above the stack is biased by a positive voltage such that the ionization from charged particles drifts away from the GEM holes to keep the detector insensitive (i.e. "blind") to signals from charged hadrons. Details about HBD photodetectors can be found in [5, 7, 9, 10]. 
The inner surface of the panels is covered by a single Kapton film with 1152 hexagonal pads printed on it. This film also serves as an additional gas seal. Each pad has an area of $6.3 \mathrm{~cm}^{2}$ and is connected to a charge sensitive amplifier on the FEE board. The size of the pad is slightly smaller than the spread of the Cherenkov photons emitted by a single particle and larger than the size of a single avalanche in the GEM. It makes the Cherenkov signal appear in several adjacent pads, while an ionizing particle produces signal primarily in a single pad.

\section{Full scale prototype}

A full scale prototype of the HBD with one instrumented sector was installed in PHENIX during the $p+p$ physics run in 2006 [12]. The magnetic field in the PHENIX was switched off and a special trigger was set up to increase the electron sample in the active area.
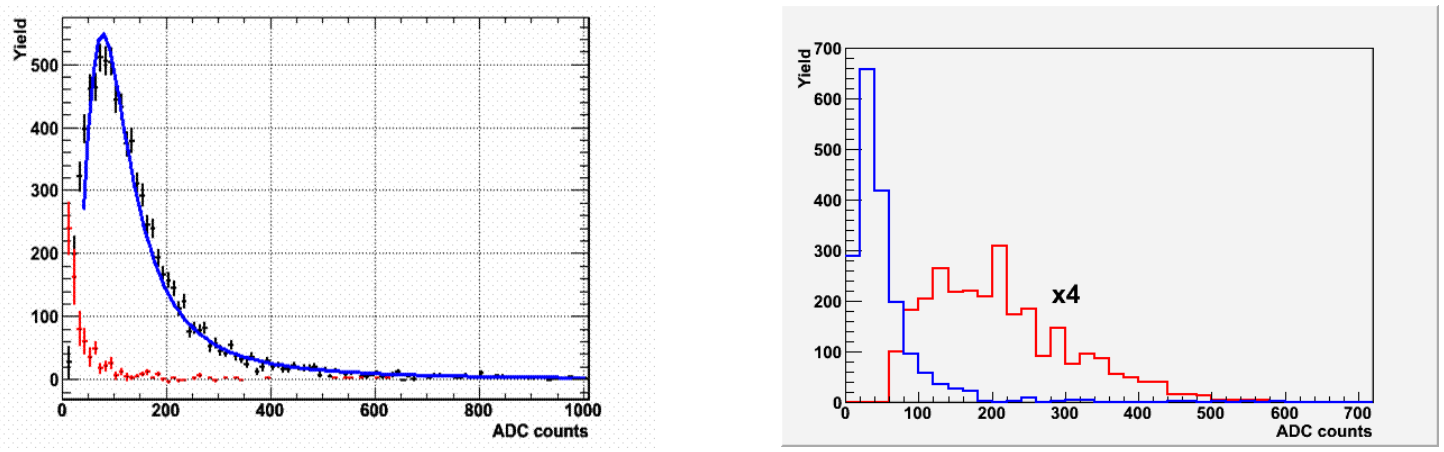

Figure 2. Left: The cluster amplitude measured with the forward (collecting) voltage bias are shown in blue (color online) and reversed bias in red. Right: all tracks (blue) and electrons (red) measured in the reversed bias configuration.

The cluster amplitude distribution produced by all particles measured with a forward (collecting) voltage bias between the mesh and the GEM is shown in the left panel of the Fig. 2. The distribution follows the Landau shape characteristic of minimum ionizing particle signals. The same distribution measured in the reversed bias configuration has much lower amplitudes and is shown with red points. In the right panel the same distribution measured with the reversed bias configuration is compared to a distribution produced by particles which are identified as electrons by the PHENIX electron identification. Signals from ionizing particles have significantly lower amplitudes than signals from electrons shown in red. Electrons can be separated from hadrons by a cut at around 100 ADC channels. The average cluster size for hadrons and electrons is also different. It was found to be 1.2 pads and 3 pads respectively.

\section{The final detector}

Both arms of the HBD were installed in PHENIX by the end of 2006. For the construction of the photodetectors 133 GEMs (85 standard plus 48 gold plated) were 
used. $65+37$ GEMs passed the initial tests. These GEMs were measured for gas gain uniformity and $48+24$ were selected and combined to minimize the gas gain variation across the surface of the triple GEM stacks. The gas gain uniformity was measured to be between $5 \%$ and $20 \%$ in all 24 modules. The CsI photocathodes were simultaneously evaporated on 4 gold-plated GEMs using the evaporator built by the INFN group and the group of Istituto Superiori di Sanita, Rome [6]. The quantum efficiency was measured and found to be the same as in the R\&D studies [7, 9, 11] and uniform within better than $5 \%$ across the area of the GEM.

During tests of the GEM stack with ${ }^{55} \mathrm{Fe}$ source we measured the dependence of the gas gain on time and rate as shown in Fig. 3 for different exposure rates.

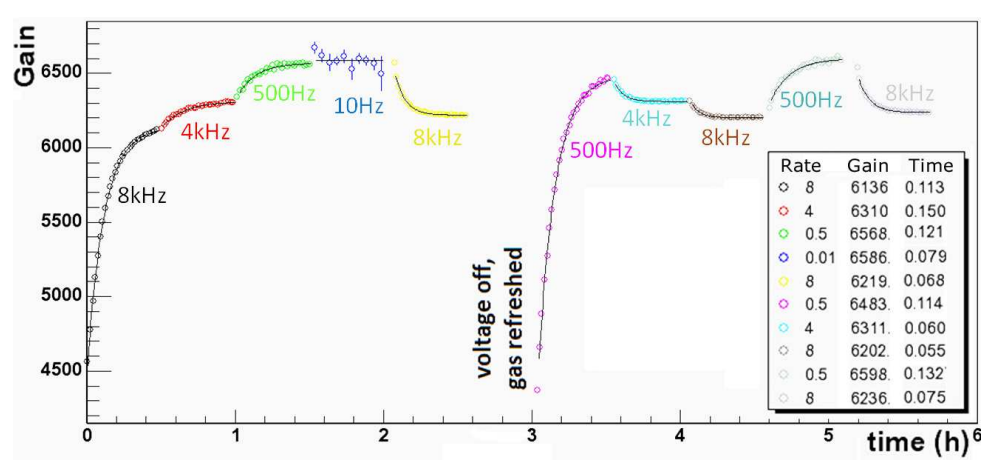

Figure 3. Gain of the GEM stack exposed to different rates of the ${ }^{55} \mathrm{Fe}$ source vs. time.
The HBD operation conditions in $\mathrm{Au}-\mathrm{Au}$ run of $\mathrm{RHIC}$ are equivalent to a source rate below $100 \mathrm{~s}^{-1}$ which impose no problem to the detector operation. However, the initial increase of gain with time may require more than one hour before stable gain conditions are established. The GEM stacks have to be under the operation voltage well before the data taking begins.

The authors acknowledge support from the Department of Energy, NSF (U.S.A.) Israel Science Foundation, US-Israel BSF and the Nella and Leon Benoziyo Center of High Energy Physics Research. The work of one of us (A.M.) is supported by the Goldhaber Fellowship at BNL with funds provided by Brookhaven Science Associates.

\section{References}

[1] K. Adcox, et al, NIM A499, (2003) 469-479

[2] A. Toia et al, nucl-ex/0510006

[3] A. Agakichev, et al, Phys. Rev. Letters 75, (1995) 1272; Phys. Letters B422, (1998) 405.

[4] M. Floris et al, nucl-ex/0606023

[5] Z. Fraenkel, et al, www.phenix.bnl.gov/WWW/TPCHBD/Weizmann_HBD_Proposal.ps

[6] Evaporation facility provided by the INFN and Istituto Superiori di Sanita in Rome, Italy, courtesy of Prof. F.Garibaldi and collaborators at Jefferson Lab.

[7] A. Kozlov, et al, NIM A523, (2004) 345

[8] Z. Fraenkel, et al, NIM A546, (2005) 466-480

[9] I. Tserruya, NIM A563, (2006) 333

[10] A. Milov et al, nucl-ex/0609014

[11] I. Ravinovich et al, Nucl. Phys A774, (2006) 903-906

[12] C. Woody et al, 2006 IEEE NSS/MIC, Oct29-Nov4, 2006, San Diego, CA. proceedings 